Bull. Korean Math. Soc. 49 (2012), No. 4, pp. 749-759

http://dx.doi.org/10.4134/BKMS.2012.49.4.749

\title{
A PROXIMAL POINT-TYPE ALGORITHM FOR PSEUDOMONOTONE EQUILIBRIUM PROBLEMS
}

\author{
Jong Kyu Kim, Pham Ngoc Anh, and Ho Geun Hyun
}

\begin{abstract}
A globally convergent algorithm for solving equilibrium problems is proposed. The algorithm is based on a proximal point algorithm (shortly $(P P A)$ ) with a positive definite matrix $M$ which is not necessarily symmetric. The proximal function in existing $(P P A)$ usually is the gradient of a quadratic function, namely, $\nabla\left(\|x\|_{M}^{2}\right)$. This leads to a proximal point-type algorithm. We first solve pseudomonotone equilibrium problems without Lipschitzian assumption and prove the convergence of algorithms. Next, we couple this technique with the Banach contraction method for multivalued variational inequalities. Finally some computational results are given.
\end{abstract}

\section{Introduction}

Let $C$ be a nonempty closed convex subset in a real Euclidean space $\mathbb{R}^{n}$ and $f: C \times C \rightarrow \mathbb{R}$ be a bifunction such that $f(x, x)=0$ for every $x \in C$. We consider the following equilibrium problem (shortly $\operatorname{EP}(f, C)$ ): Find $x^{*} \in C$ such that

$$
f\left(x^{*}, y\right) \geq 0 \quad \forall y \in C .
$$

For the main results of this paper, we assume that:

(A.1) $f(\cdot, \cdot)$ is pseudomonotone on $C$,

(A.2) $f(x, \cdot)$ is convex on $C$ for all $x \in C$,

(A.3) $f$ is continuous on $C \times C$,

(A.4) The solution set $S$ of $E P(f, C)$ is nonempty,

(A.5) The matrix $M$ is positive definite.

Equilibrium problems appear frequently in many practical problems arising, for instance, physics, engineering, game theory, transportation, economics and network (see $[10,13])$. They become an attractive field for many researchers both in theory and applications (see $[2,3,5,9,15,16,17])$. These problems are models whose formulation includes optimization, variational inequalities, (vector) optimization problems, fixed point problems, saddle point problems,

Received March 28, 2011; Revised May 3, 2011.

2010 Mathematics Subject Classification. 65K10, 90C25.

Key words and phrases. equilibrium problems, proximal point algorithm, pseudomonotonicity, linear proximal function, Banach contraction method. 
Nash equilibria and complementarity problems as particular cases (see [8, 9, 10]).

There exist several methods for solving $\operatorname{EP}(f, C)$ with monotone bifunction $f$. We can name as projection methods (see [10]) or the interior quadratic regularization methods (see $[2,3]$ ). The interior quadratic regularization technique has been used to develop proximal iterative algorithm for variational inequalities (see [1, 7]). In [4], the authors proposed a cutting hyperplane method for pseudomonotone equilibrium problems. The first we construct an appropriate hyperplane which separates the current iterative point from the solution set of the problem. The next iterate is obtained as the projection of the current iterative point onto the intersection of the feasible set with the halfspace containing the solution set (see [12]).

If the bifunction $f$ is defined by

$$
f(x, y):=\sup \{\langle w, y-x\rangle \mid w \in F(x)\},
$$

where $F: C \rightarrow 2^{\mathbb{R}^{n}}$ is a multivalued mapping such that $F(x) \neq \emptyset$ for all $x \in C$, then $\operatorname{EP}(f, C)$ can be formulated as the multivalued variational inequalities (shortly $(M V I))$ : Find $x^{*} \in C, w^{*} \in F\left(x^{*}\right)$ such that

$$
\left\langle w^{*}, x-x^{*}\right\rangle \geq 0 \quad \forall x \in C \text {. }
$$

A classical method for solving $(M V I)$ is the proximal point algorithm (see $[13,16])$. For given $x^{k} \in C$, the new iterate $x^{k+1}$ generated by $(P P A)$ is the unique solution to the following auxiliary multivalued variational inequality: Find $x^{0} \in C, w^{0} \in F\left(x^{0}\right)$ such that

$$
\left\langle w^{0}+M\left(x-x^{k}\right), y-x^{0}\right\rangle \geq 0 \quad \forall y \in C,
$$

where $M$ is a symmetric positive-definite matrix and $M\left(x-x^{k}\right)$ is called the proximal function in $(P P A)$. This linear proximal function is the gradient of a quadratic function, namely,

$$
M\left(x-x^{k}\right)=\nabla\left(\frac{1}{2}\left\|x-x^{k}\right\|_{M}^{2}\right) .
$$

Recently, a number of authors have concentrated on the generalization of $(P P A)$ by replacing the linear proximal function $M\left(x-x^{k}\right)$ with some nonlinear functions $d\left(x, x^{k}\right)$ arising from appropriately formular Bregman functions (see $[7,11])$.

In this paper, we consider propose the (PPA)-type method for solving $E P(f, C)$ with a positive definite matrix $M$ which is not necessarily symmetric and a pseudomonotone mapping $f$ which is not assumed to be Lipschitz on $C$. In Section 3, we prove the convergence theorem. In Section 4, we couple the proximal point algorithm with the Banach contraction method for solving $(M V I)$ and present some numerical experiences to illustrate the behavior of the proposed algorithms. 


\section{Proposed methods}

We give some well known definitions which will be required in our following analysis.

Definition 2.1. A bifunction $f: C \times C \rightarrow \mathbb{R} \cup\{+\infty\}$ is said to be

(i) $\gamma$-strongly monotone on $C$ if for each $x, y \in C$,

$$
f(x, y)+f(y, x) \leq-\gamma\|x-y\|^{2} ;
$$

(ii) monotone on $C$ if for each $x, y \in C$,

$$
f(x, y)+f(y, x) \leq 0 ;
$$

(iii) pseudomonotone on $C$ if for each $x, y \in C$,

$$
f(x, y) \geq 0 \Rightarrow f(y, x) \leq 0 .
$$

It is observe that (i) $\Rightarrow$ (ii) $\Rightarrow$ (iii). However, if $f$ is pseudomonotone, then $f$ need not be monotone on $C$. There are some examples in [18].

Now we define the projection under the Euclidean norm. Let $C$ be a closed convex subset in $\mathbb{R}^{n}$, we define the projection on $C$ by $\operatorname{Pr}_{C}(\cdot)$, i.e.,

$$
\operatorname{Pr}_{C}(x)=\operatorname{argmin}\{\|y-x\| \mid y \in C\} \quad \forall x \in \mathbb{R}^{n} .
$$

Algorithm 2.2. Step 0 : Given an $(n \times n)$ positive definite matrix $M, \varepsilon>$ $0, x^{0} \in C$. Set $k=0$.

Step 1 : Find a proximal point $\bar{x}^{k}$ which is the solution of the following $\left(\right.$ shortly $\left.P_{k}\right)$ : Find $\bar{x} \in C$, such that

$$
f\left(x^{k}, y\right)+\left\langle M\left(\bar{x}-x^{k}\right), y-\bar{x}\right\rangle \geq 0 \quad \forall y \in C .
$$

If $\left\|x^{k}-\bar{x}^{k}\right\|<\epsilon$, then terminate. Otherwise, go to Step 2 .

Step 2 : Compute

$$
x^{k+1}=x^{k}-\alpha_{k} M\left(x^{k}-\bar{x}^{k}\right),
$$

with

$$
\alpha_{k}=\gamma \frac{\left\langle M\left(x^{k}-\bar{x}^{k}\right), x^{k}-\bar{x}^{k}\right\rangle}{\left\|M\left(x^{k}-\bar{x}^{k}\right)\right\|^{2}}, \gamma \in[1,2) .
$$

Increase $k$ by 1 and go to Step 1.

Remark 2.3. The problem (2.1) in Step 1 is similar to the problem (1.1) of classical $(P P A)$, the only difference is that matrix $M$ in $(2.1)$ is positive definite which is not necessarily symmetric. Thus, the proposed method is a general form of $(P P A)$-based methods with a linear proximal function. Note that we can not take $\bar{x}^{k}$ as the new iteration unless $M$ is symmetric and positive definite.

Remark 2.4. In comparison with solving problem (2.1), the additional computational load for updating $x^{k+1}$ via (2.2)-(2.3) is slight. 
The solution of $(2.1), \bar{x}^{k}$ is the proximal point in the $k$-th iteration. It is viewed as a test vector because it is a solution of $E P(f, C)$ if and only if $x^{k}=\bar{x}^{k}$. In fact, $\left\|x^{k}-\bar{x}^{k}\right\|$ can be viewed as an error bound function which measures how much $\bar{x}^{k}$ fails to be a solution point of MVIP. Therefore, we have the following.

Proposition 2.5. In Step 1, if $\left\|x^{k}-\bar{x}^{k}\right\| \leq \epsilon$, then $x^{k}$ is an $\epsilon$-solution to $E P(f, C)$.

The following lemma gives important tool inequalities which are useful in the next discussion (see [13]).

Lemma 2.6. For any $x, y \in \mathbb{R}^{n}$, the mapping $P_{C}$ satisfies

$$
\begin{aligned}
& \left\langle P_{C}(x)-x, y-P_{C}(x)\right\rangle \geq 0, \quad \forall y \in C, \\
& \left\|P_{C}(x)-y\right\|^{2} \leq\|x-y\|^{2}-\left\|x-P_{C}(x)\right\|^{2}, \quad \forall y \in C .
\end{aligned}
$$

\section{Convergence analysis of the proposed method}

Note that, for any $x^{*} \in S,\left(x^{k}-x^{*}\right)$ is the gradient of the unknown distance function $\frac{1}{2}\left\|x-x^{*}\right\|^{2}$ at point $x^{k} \notin S$. A point $d$ is called a descent derection of $\frac{1}{2}\left\|x-x^{*}\right\|^{2}$ at the point $x^{k}$ if

$$
\left\langle x^{k}-x^{*}, d\right\rangle<0 .
$$

This subsection shows that $-M\left(x^{k}-\bar{x}^{k}\right)$ is a descent direction of the unknown distance function $\frac{1}{2}\left\|x-x^{*}\right\|^{2}$ at the point $x^{k}$ (see [14]).

Lemma 3.1. Let $\bar{x}^{k}$ be the proximal point generated by (2.1) from given $x^{k} \in$ $C$. Then, for any $x^{*} \in S$ we have

$$
\left\langle M\left(x^{k}-\bar{x}^{k}\right), x^{k}-x^{*}\right\rangle \geq\left\langle M\left(x^{k}-\bar{x}^{k}\right), x^{k}-\bar{x}^{k}\right\rangle .
$$

Proof. Since $\bar{x}^{k} \in C$ is a solution of (2.1), we have

$$
f\left(\bar{x}^{k}, y\right)+\left\langle M\left(\bar{x}^{k}-x^{k}\right), y-\bar{x}^{k}\right\rangle \geq 0 \quad \forall y \in C .
$$

Substituting $x^{*} \in C$ into this, we obtain

$$
f\left(\bar{x}^{k}, x^{*}\right)+\left\langle M\left(\bar{x}^{k}-x^{k}\right), x^{*}-\bar{x}^{k}\right\rangle \geq 0 .
$$

On the other hand, $x^{*} \in S$ follows that

$$
f\left(x^{*}, y\right) \geq 0 \quad \forall y \in C .
$$

Since $\bar{x}^{k} \in C$ and $f$ is pseudomonotone on $C$, we have

$$
f\left(\bar{x}^{k}, x^{*}\right) \leq 0 \quad \forall y \in C .
$$

Combining (3.2) and (3.3), we have

$$
\left\langle M\left(\bar{x}^{k}-x^{k}\right), x^{*}-\bar{x}^{k}\right\rangle \geq 0 .
$$

Hence

$$
\left\langle M\left(x^{k}-\bar{x}^{k}\right), x^{k}-x^{*}\right\rangle \geq\left\langle M\left(x^{k}-\bar{x}^{k}\right), x^{k}-\bar{x}^{k}\right\rangle .
$$


Since the matrix $M$ is positive definite, we have

$$
\left\langle M\left(x^{k}-\bar{x}^{k}\right), x^{k}-\bar{x}^{k}\right\rangle \geq \tau\left\|x^{k}-\bar{x}^{k}\right\|^{2} .
$$

Hence, Lemma 3.1 shows that $-M\left(x^{k}-\bar{x}^{k}\right)$ is a descent direction of $\frac{1}{2}\left\|x-\bar{x}^{k}\right\|^{2}$ at the point $x^{k} \notin S$.

Remark 3.2. If the matrix $M$ in (2.1) is symmetric, then it follows from (3.1) that

$$
\begin{aligned}
\left\|\bar{x}^{k}-x^{*}\right\|_{M}^{2} & =\left\|\left(x^{k}-x^{*}\right)-\left(x^{k}-\bar{x}^{k}\right)\right\|_{M}^{2} \\
& =\left\|x^{k}-x^{*}\right\|_{M}^{2}-2\left\langle M\left(x^{k}-\bar{x}^{k}\right), x^{k}-x^{*}\right\rangle+\left\|x^{k}-\bar{x}^{k}\right\|_{M}^{2} \\
& \leq\left\|x^{k}-x^{*}\right\|_{M}^{2}-\left\|x^{k}-\bar{x}^{k}\right\|_{M}^{2} .
\end{aligned}
$$

Therefore, we can directly set $x^{k+1}:=\bar{x}^{k}$ as the new iteration and the convergence follows from the above inequality. However, in the case that $M$ is asymmetric, we cannot establish the convergence when we directly set $x^{k+1}:=$ $\bar{x}^{k}$ as the new iteration. In our proposed method, the new iteration $x^{k+1}$ is updated by (2.2), the computational load for updating $x^{k+1}$ in Step 2 is small.

In order to explain why we have the optimal step $\alpha_{k}^{*}$ as defined in (2.3), instead of the updating formula (2.2), we define the stepsize dependent new iterations by

$$
x^{k+1}=x^{k}-\alpha M\left(x^{k}-\bar{x}^{k}\right) .
$$

In this way,

$$
\theta(\alpha):=\left\|x^{k}-x^{*}\right\|^{2}-\left\|x^{k+1}(\alpha)-x^{*}\right\|^{2}
$$

is the profit function in the $k$-th iteration by using the updating form (3.4). Since $x^{*}$ is the solution point and thus is unknown, we cannot maximize $\theta(\alpha)$ directly. The following theorem introduces a tight lower bound of $\theta(\alpha)$, namely $q(\alpha)$, which does not include the unknown solution $x^{*}$.

Theorem 3.3. For any $x^{*} \in S$ and $\alpha \geq 0$, we have

$$
\theta(\alpha) \geq q(\alpha)
$$

where

$$
q(\alpha)=2 \alpha\left\langle M\left(x^{k}-\bar{x}^{k}\right), x^{k}-\bar{x}^{k}\right\rangle-\alpha^{2}\left\|M\left(x^{k}-\bar{x}^{k}\right)\right\|^{2} .
$$

Proof. Combining (3.4), (3.5), (3.6) and (3.7), we get

$$
\begin{aligned}
\theta(\alpha) & =\left\|x^{k}-x^{*}\right\|^{2}-\left\|\left(x^{k}-x^{*}\right)-\alpha M\left(x^{k}-\bar{x}^{k}\right)\right\|^{2} \\
& =2\left\langle M\left(x^{k}-\bar{x}^{k}\right), x^{k}-x^{*}\right\rangle-\alpha^{2}\left\|M\left(x^{k}-\bar{x}^{k}\right)\right\|^{2} \\
& \leq 2\left\langle M\left(x^{k}-\bar{x}^{k}\right), x^{k}-\bar{x}^{k}\right\rangle-\alpha^{2}\left\|M\left(x^{k}-\bar{x}^{k}\right)\right\|^{2} \\
& =q(\alpha) .
\end{aligned}
$$


Noting that $q(\alpha)$ is a quadratic function of $\alpha$ and thus it reaches its maximum at

$$
\alpha_{k}^{*}=\frac{\left\langle M\left(x^{k}-\bar{x}^{k}\right), x^{k}-\bar{x}^{k}\right\rangle}{\left\|M\left(x^{k}-\bar{x}^{k}\right)\right\|^{2}},
$$

this is just the same defined in (2.3). Because an inequality is used in proof of (3.6), in practical computation, taking a relaxed factor $\gamma \geq 1$ is good for fast convergence. Note that for any $\alpha_{k}=\gamma \alpha_{k}^{*}$, it follows from (3.6), (3.7) and (2.3) that

$$
\theta\left(\gamma \alpha_{k}^{*}\right) \geq q\left(\gamma \alpha_{k}^{*}\right)=\gamma(2-\gamma) \alpha_{k}^{*}\left\langle M\left(x^{k}-\bar{x}^{k}\right), x^{k}-\bar{x}^{k}\right\rangle .
$$

In order to guarantee that the right-hand side of (3.8) is positive, we take $\gamma \in[1,2)$.

The following theorem point out that a relation between the sequence $\left\{x^{k}\right\}$ generated by the proposed method and any solution of problems $(M V I)$.

Theorem 3.4. For any $x^{*} \in S$, the sequence $\left\{x^{k}\right\}$ generated by the proposed method satisfies

$$
\left\|x^{k+1}-x^{*}\right\|^{2} \leq\left\|x^{k}-x^{*}\right\|^{2}-c_{0}\left\|x^{k}-\bar{x}^{k}\right\|^{2},
$$

where $c_{0}>0$ is a constant.

Proof. First, it follows from (3.5) and (3.8) that

$$
\left\|x^{k}-x^{*}\right\|^{2}-\left\|x^{k+1}-x^{*}\right\|^{2} \geq \gamma(2-\gamma) \alpha_{k}^{*}\left\langle M\left(x^{k}-\bar{x}^{k}\right), x^{k}-\bar{x}^{k}\right\rangle .
$$

Thus

$$
\left\|x^{k+1}-x^{*}\right\|^{2} \leq\left\|x^{k}-x^{*}\right\|^{2}-\gamma(2-\gamma) \alpha_{k}^{*}\left\langle M\left(x^{k}-\bar{x}^{k}\right), x^{k}-\bar{x}^{k}\right\rangle .
$$

Since

$$
\left\langle M\left(x^{k}-\bar{x}^{k}\right), x^{k}-\bar{x}^{k}\right\rangle \geq \tau\left\|x^{k}-\bar{x}^{k}\right\|^{2},
$$

we have

$$
\begin{aligned}
\alpha_{k}^{*} & =\frac{\left\langle M\left(x^{k}-\bar{x}^{k}\right), x^{k}-\bar{x}^{k}\right\rangle}{\left\|M\left(x^{k}-\bar{x}^{k}\right)\right\|^{2}} \\
& \geq \frac{\tau}{\left\|M^{T} M\right\|} .
\end{aligned}
$$

Combining this with (3.11), we get

$$
\alpha_{k}^{*}\left\langle M\left(x^{k}-\bar{x}^{k}\right), x^{k}-\bar{x}^{k}\right\rangle \geq \frac{\tau^{2}}{\left\|M^{T} M\right\|}\left\|x^{k}-\bar{x}^{k}\right\|^{2} .
$$

Replacing (3.12) to (3.10) and by setting

$$
c_{0}=\frac{\gamma(2-\gamma) \tau^{2}}{\left\|M^{T} M\right\|}
$$

which prove (3.9).

The following theorem shows the convergence of the sequence $\left\{x^{k}\right\}$. 
Theorem 3.5. The sequence $\left\{x^{k}\right\}$ generated by the proposed method converges to some $x^{\infty}$ which is a solution point of $E P(f, C)$.

Proof. It follows from (3.9) that the sequence $\left\{\left\|x^{k}-x^{*}\right\|\right\}$ is nonincreasing. Since it is bounded below by 0 , it must be convergent. Thus, the sequence $\left\{x^{k}\right\}$ is bounded. Let $x^{\infty}$ be a cluster point of $\bar{x}^{k}$. By Bolzano-Weierstrass theorem, there exists subsequence $\bar{x}^{k_{j}}$ of $\bar{x}^{k}$ such that $\bar{x}^{k_{j}} \rightarrow x^{\infty}$ as $j \rightarrow \infty$. For every $\bar{x}^{k_{j}} \in C$, we have

$$
f\left(x^{k_{j}}, x\right)+\left\langle M\left(\bar{x}^{k_{j}}-x^{k_{j}}\right), x-\bar{x}^{k_{j}}\right\rangle \geq 0 \quad \forall x \in C .
$$

From the continuity of $f$ on $C$ and

$$
\lim _{j \rightarrow \infty}\left\|x^{k_{j}}-\bar{x}^{k_{j}}\right\|=0
$$

we get

$$
x^{\infty} \in C, \quad f(\bar{x}, x) \geq 0 \quad \forall x \in C,
$$

and thus $x^{\infty}$ is a solution point. Note that, inequality (3.9) is true for the solution point of $E P(f, C)$, hence

$$
\left\|x^{k+1}-x^{\infty}\right\|^{2} \leq\left\|x^{k}-x^{\infty}\right\|^{2} \quad \forall k \geq 0,
$$

and the sequence $\left\{x^{k}\right\}$ converges to $x^{\infty}$.

\section{Application to the Banach contraction algorithm for (MVI)}

In this section, we will combine Algorithm 2.1 with the Banach contraction method for solving the variational inequalities $(M V I)$, where the monotone cost operator is Lipschitz on $C$. The auxiliary problems $P_{k}$ are strongly monotone variational inequalities with constant $\|M\|$. Then these can be solved the efficiency by the Banach contraction algorithm. For every $x \in C, k=0,1,2, \ldots$, we denote $F_{k}(x):=F(x)+M\left(x-x^{k}\right)$.

Algorithm 4.1. Step 0 : Given an $(n \times n)$ positive definite matrix $M, \varepsilon>$ $0, x^{0} \in C, w^{0} \in F\left(x^{0}\right)$. Set $k=0$. Choose $\beta>\frac{L^{2}}{2\|M\|}$.

Step 1 : Iteration $j=0,1,2, \ldots$ Choose $x^{k, 0}=x^{0}, w^{k, 0} \in F_{k}\left(x^{k, 0}\right)$ and solve the strongly convex program

$$
x^{k, j}:=\operatorname{argmin}\left\{\frac{1}{2} \beta\left\|x-x^{k, j}\right\|^{2}+\left\langle w^{k, j}, x-x^{k, j}\right\rangle \mid x \in C\right\}
$$

to obtain its unique solution $x^{k, j+1}$. If $x^{k, j+1}=x^{k, j}$, then $\bar{x}^{k}:=x^{k, j}, \bar{w}^{k}=$ $w^{k, j}$. Otherwise, choose $w^{k, j+1} \in F\left(x^{k, j+1}\right)$.

Increase $j$ by 1 and go to Iteration $j$. If $\bar{x}^{k}=x^{k}$, then stop. Otherwise, go to Step 2.

Step 2 : Compute

$$
w^{k}=P_{F\left(x^{k}\right)}\left(\bar{w}^{k}\right), x^{k+1}=x^{k}-\alpha_{k} M\left(x^{k}-\bar{x}^{k}\right),
$$

with

$$
\alpha_{k}=\gamma \frac{\left\langle M\left(x^{k}-\bar{x}^{k}\right), x^{k}-\bar{x}^{k}\right\rangle}{\left\|M\left(x^{k}-\bar{x}^{k}\right)\right\|^{2}}, \gamma \in[1,2) \text {. }
$$


Increase $k$ by 1 and go to Step 1.

The convergence of the sequence $\left\{x^{k, j}\right\}_{j=1}^{\infty}$ of the strongly monotone variational inequalities $\left(V I P_{k}\right)$ is defined by the following proposition.

Proposition 4.2 (see Theorem 3.1 in [6]). Suppose that F is L-Lipschitz on C. If Algorithm 3.1 terminates at Iteration $j$ in Step 1 , then $\left(x^{k}, w^{k}\right)$ is a solution to $\left(V I P_{k}\right)$. Moreover, if $\left(x^{k, *}, w^{k, *}\right)$ is a solution to $\left(V I P_{k}\right)$, then we have

$$
\left\|x^{k, j}-x^{k, *}\right\| \leq \frac{\delta^{j}}{1-\delta^{j}}\left\|x^{k, 1}-x^{k, 0}\right\| \quad \forall j=1,2, \ldots,
$$

and every cluster point $\bar{w}^{k, *}$ of the sequence $\left\{w^{k, j}\right\}$ satisfies $\bar{w}^{k, *} \in F\left(x^{k, *}\right)$, where

$$
\delta:=\sqrt{1-\frac{2 \beta}{\|M\|}+\frac{L^{2}}{\|M\|^{2}}} .
$$

Proposition 4.2 shows that Algorithm 3.1 terminates at Step 1. It means that $x^{k, j}$ is a solution to $\left(V I P_{k}\right)$. By Theorem 3.3 , we have the following convergence results of Algorithm 4.1.

Theorem 4.3. The sequences $\left\{x^{k}\right\}$ and $\left\{w^{k}\right\}$ generated by the proposed method converge to $\left(x^{\infty}, w^{\infty}\right)$ which is a solution of problems $(M V I)$.

Now we illustrate our Algorithms with the oligopolistic market equilibrium model considered in [13]. Assume that there are $n$ firms supplying a homogeneous product and that the price $p$ depends on its quantity $\sigma_{x}=$ $x_{1}+x_{1}+\cdots+x_{n}$, i.e., $p=p\left(\sigma_{x}\right)$. Let $h_{i}\left(x_{i}\right)$ denote the total cost to the firm $i$ for supplying $x_{i}$ units of the product. Then, the profit of the firm $i$ is $x_{i} p\left(\sigma_{x}\right)-h_{i}\left(x_{i}\right)$. Naturally, each firm seeks to maximize its own profit by choosing the corresponding production level. Suppose that the strategy set $C$ is a polyhedral convex subset in $\mathbb{R}^{n}$ given by

$$
C:=\left\{x \in \mathbb{R}^{n} \mid 13 \leq \sum_{i=1}^{n} x_{i} \leq 25,1 \leq x_{i} \leq 5 \quad i=1,2, \ldots, n\right\} .
$$

Thus, the oligopolistic market equilibrium problem can be formulated as a Nash equilibrium noncooperative game, where the $i$ th player has the strategy set $\mathrm{C}$ and the utility function

$$
f_{i}\left(x_{1}, \ldots, x_{n}\right)=x_{i} p\left(\sum_{i=1}^{n}\right)-h_{i}\left(x_{i}\right) \quad i=1,2, \ldots, n .
$$

As usual, a point $x^{*}=\left(x_{1}^{*}, \ldots, x_{n}^{*}\right) \in C$ is said to be an equilibrium point for this problem if

$$
f_{i}\left(x_{1}^{*}, \ldots, x_{i-1}^{*}, y_{i}, x_{i+1}^{*}, \ldots, x_{n}^{*}\right) \leq f_{i}\left(x_{1}^{*}, \ldots, x_{n}^{*}\right) \quad \forall i=1,2, \ldots, n .
$$


Proposition 4.4 (see [13]). A point $x^{*}$ is an equilibrium point for the oligopolistic market problem if and only if it is a solution to $(M V I)$, where $C$ is the polyhedral given by (4.1) and $F(x)=H(x)-p\left(\sigma_{x}\right) e-p^{\prime}\left(\sigma_{x}\right) x$, where $H(x)=$ $\left(h_{1}^{\prime}\left(x_{1}\right), \ldots, h_{n}^{\prime}\left(x_{n}\right)\right)^{T}, e=(1, \ldots, 1)^{T}, \sigma_{x}=\langle x, e\rangle$.

Proposition 4.5 (see [13]). Let $p: C \rightarrow \mathbb{R}_{+}$be convex, twice continuously differentiable, and nonincreasing, let the function $\mu_{\tau}: \mathbb{R}_{+} \rightarrow \mathbb{R}_{+}$, defined by $\mu_{\tau}\left(\sigma_{x}\right)=\sigma_{x} p\left(\sigma_{x}+\tau\right)$ be concave for every $\tau \geq 0$. Also, let the function $h_{i}: \mathbb{R}_{+} \rightarrow \mathbb{R}, i=1, \ldots, n$, be convex and twice continuously differentiable. Then, the cost mapping $F(x)=H(x)-p\left(\sigma_{x}\right) e-p^{\prime}\left(\sigma_{x}\right) x$ is monotone on $C$.

It is very easy to see that the cost mapping $F$ is Lipschitz on $C$ with Lipschitz constant $L<1$. In this example, we choose (randomly generalized)

$$
\begin{aligned}
n & :=7, \\
H(x) & :=\left(2 x_{1}+1,3 x_{2}+4,4 x_{3}+2,1.5 x_{4}+3,4 x_{5}+1, x_{6}-2,3 x_{7}+1\right)^{T}, \\
p(t) & :=\frac{2}{3 t} t \in(0,+\infty), \\
x^{0} & :=(1.9,1,1,1,1,5,1)^{T} \in C,
\end{aligned}
$$

The tolerance $\epsilon=10^{-6}, \gamma=\frac{3}{2}$,

$$
M=\left[\begin{array}{ccccccc}
1 & 0 & 0 & 0 & 0 & 0 & 0 \\
0 & 2 & 0 & 0 & 0 & 0 & 1 \\
0 & 0 & 3 & 0 & 0 & 0 & 4 \\
0 & 0 & 0 & 1.5 & 0 & 0 & 3 \\
0 & 0 & 0 & 0 & 2 & 0 & 2 \\
0 & 0 & 0 & 0 & 0 & 1.6 & 2 \\
0 & 0 & 0 & 0 & 1 & 0 & 2
\end{array}\right]_{7 \times 7} .
$$

Then, the eigenvalues of the matrix $M$ are: $2,3,1.5,1.6,3.4142,0.5858,1$, the norm of $M$ is $\|M\|=6.6248$ and $\beta>\frac{L^{2}}{2\|M\|} \approx 0.755$. If we choose $\beta=1$, then $\delta \approx 0.7209$. In this case, we obtained the following iterations.

\begin{tabular}{cccccccc}
\hline Iter $(k)$ & $x_{1}^{k}$ & $x_{2}^{k}$ & $x_{3}^{k}$ & $x_{4}^{k}$ & $x_{5}^{k}$ & $x_{6}^{k}$ & $x_{7}^{k}$ \\
\hline 0 & 1.9 & 1 & 1 & 1 & 1 & 5 & 1 \\
1 & 2.0475 & 1.0417 & 1.0246 & 1.6452 & 1.1338 & 5.0954 & 1.4095 \\
2 & 2.0364 & 0.9797 & 0.9285 & 1.5003 & 1.0200 & 4.9796 & 1.3394 \\
3 & 2.0558 & 1.0043 & 1.0409 & 1.5263 & 1.0573 & 5.0158 & 1.3717 \\
4 & 2.0898 & 1.0170 & 0.9878 & 1.5089 & 1.0774 & 5.0317 & 1.4046 \\
5 & 2.0813 & 0.9914 & 0.9858 & 1.4641 & 1.0368 & 4.9905 & 1.3784 \\
6 & 2.0864 & 0.9997 & 1.0128 & 1.4741 & 1.0490 & 5.0025 & 1.3887 \\
7 & 2.0903 & 1.0034 & 1.0072 & 1.4761 & 1.0543 & 5.0074 & 1.3948 \\
8 & 2.0906 & 0.9983 & 0.9919 & 1.4641 & 1.0462 & 4.9989 & 1.3917 \\
9 & 2.0920 & 1.0005 & 1.0036 & 1.4664 & 1.0493 & 5.0019 & 1.3946 \\
10 & 2.0943 & 1.0017 & 0.9991 & 1.4648 & 1.0509 & 5.0029 & 1.3979 \\
11 & 2.0933 & 0.9993 & 0.9982 & 1.4608 & 1.0472 & 4.9992 & 1.3955 \\
12 & 2.0938 & 1.0000 & 1.0011 & 1.4617 & 1.0483 & 5.0003 & 1.3964 \\
13 & 2.0940 & 1.0003 & 1.0003 & 1.4619 & 1.0487 & 5.0007 & 1.3970 \\
14 & 2.0939 & 0.9998 & 0.9996 & 1.4608 & 1.0479 & 4.9998 & 1.3965 \\
15 & 2.0940 & 1.0000 & 1.0003 & 1.4610 & 1.0482 & 5.0001 & 1.3968 \\
\hline
\end{tabular}


The approximate solution obtained after 15 iterations is

$$
x^{15}=(2.0940,1.0000,1.0003,1.4610,1.0482,5.0001,1.3968)^{T} .
$$

Acknowledgment. This work was completed while the second author was staying at Kyungnam University for the NRF Postdoctoral Fellowship for Foreign Researchers. And this work was supported by Kyungnam University Research Fund 2010.

\section{References}

[1] P. N. Anh, An interior-quadratic proximal method for solving monotone generalized variational inequalities, East-West Journal of Mathematics 10 (2008), 81-100.

[2] — A logarithmic quadratic regularization method for solving pseudomonotone equilibrium problems, Acta Mathematica Vietnamica 34 (2009), 183-200.

[3] _ An LQP regularization method for equilibrium problems on polyhedral, Vietnam Journal of Mathematics 36 (2008), 209-228.

[4] P. N. Anh and J. K. Kim, A cutting hyperplane method for solving pseudomonotone nonlipschitzian equilibrium problems, Submitted, 2011.

[5] P. N. Anh, L. D. Muu, V. H. Nguyen, and J. J. Strodiot, Using the Banach contraction principle to implement the proximal point method for multivalued monotone variational inequalities, J. Optim. Theory Appl. 124 (2005), no. 2, 285-306.

[6] P. N. Anh, L. D. Muu, and J. J. Strodiot, Generalized projection method for nonLipschitz multivalued monotone variational inequalities, Acta Mathematica Vietnamica 34 (2009), 67-79.

[7] A. Auslender, M. Teboulle, and S. Bentiba, A logarithmic-quadratic proximal method for variational inequalities, Comput. Optim. Appl. 12 (1999), no. 1-3, 31-40.

[8] G. Bigi, M. Castellani, and M. Pappalardo, A new solution method for equilibrium problems, Optim. Methods Softw. 24 (2009), no. 6, 895-911.

[9] E. Blum and W. Oettli, From optimization and variational inequality to equilibrium problems, Math. Student 63 (1994), no. 1-4, 123-145.

[10] P. Daniele, F. Giannessi, and A. Maugeri, Equilibrium Problems and Variational Models, Kluwer, 2003

[11] F. Facchinei and J. S. Pang, Finite-Dimensional Variational Inequalities and Complementary Problems Vol. I, II, Springer-Verlag, New York, 2003.

[12] B. S. He, X. L. Fu, and Z. K. Jiang, Proximal-point algorithm using a linear proximal term, J. Optim. Theory Appl. 141 (2009), no. 2, 299-319.

[13] I. V. Konnov, Combined Relaxation Methods for Variational Inequalities, SpringerVerlag, Berlin, 2001

[14] O. L. Mangasarian and M. V. Solodov, A linearly convergent derivative-free descent method for strongly monotone complementarity problem, Comput. Optim. Appl. 14 (1999), no. 1, 5-16.

[15] G. Mastroeni, Gap function for equilibrium problems, J. Global Optim. 27 (2003), no. 4, 411-426.

[16] A. Moudafi, Proximal point algorithm extended to equilibrium problem, J. Nat. Geom. 15 (1999), no. 1-2, 91-100.

[17] M. A. Noor, Auxiliary principle technique for equilibrium problems, J. Optim. Theory Appl. 122 (2004), no. 2, 371-386.

[18] S. Schaible, S. Karamardian, and J. P. Crouzeix, Characterizations of generalized monotone maps, J. Optim. Theory Appl. 76 (1993), no. 3, 399-413. 
Jong KYU KIM

Department of Mathematics Education

KYUNGNAM University

MASAN 631-701, Korea

E-mail address: jongkyuk@kyungnam.ac.kr

Pham NGOC Anh

Department of Mathematics Education

KyUNGNAM UNIVERSITY

Masan 631-701, Korea

E-mail address: anhpn@ptit.edu.vn

Ho Geun Hyun

Department of Mathematics Education

KYUNGNAM UNIVERSITY

MASAN 631-701, Korea

E-mail address: hyunhg8285@kyungnam.ac.kr 